\title{
Annual Scheduling of Atlantic Fleet Naval Combatants
}

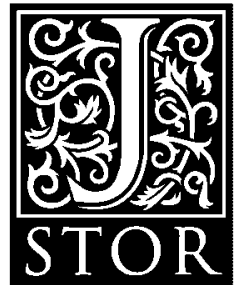

Gerald G. Brown; Clark E. Goodman; R. Kevin Wood

Operations Research, Volume 38, Issue 2 (Mar. - Apr., 1990), 249-259.

Stable URL:

http://links.jstor.org/sici?sici=0030-364X\%28199003\%2F04\%2938\%3A2\%3C249\%3AASOAFN\%3E2.0.CO\%3B2-F

Your use of the JSTOR archive indicates your acceptance of JSTOR's Terms and Conditions of Use, available at http://www.jstor.org/about/terms.html. JSTOR's Terms and Conditions of Use provides, in part, that unless you have obtained prior permission, you may not download an entire issue of a journal or multiple copies of articles, and you may use content in the JSTOR archive only for your personal, non-commercial use.

Each copy of any part of a JSTOR transmission must contain the same copyright notice that appears on the screen or printed page of such transmission.

Operations Research is published by INFORMS. Please contact the publisher for further permissions regarding the use of this work. Publisher contact information may be obtained at http://www.jstor.org/journals/informs.html.

Operations Research

(C)1990 INFORMS

JSTOR and the JSTOR logo are trademarks of JSTOR, and are Registered in the U.S. Patent and Trademark Office. For more information on JSTOR contact jstor-info@umich.edu.

(C)2003 JSTOR 


\title{
ANNUAL SCHEDULING OF ATLANTIC FLEET NAVAL COMBATANTS
}

\author{
GERALD G. BROWN, CLARK E. GOODMAN and R. KEVIN WOOD \\ Naval Postgraduate School, Monterey, California
}

(Received September 1987; revision received November 1988; accepted February 1989)

\begin{abstract}
Employment scheduling is the process whereby U. S. Navy ships, submarines, aircraft and other units are assigned to major operations, exercises, maintenance periods, inspections and other events; the employment schedule directly influences fleet combat readiness. Currently, this process is largely manual requiring several full-time scheduling officers and additional personnel at various levels of management. We introduce an optimization model that automates a substantial part of the employment scheduling problem. The model is formulated as a generalized set partitioning problem and is applied to the annual planning schedule for naval surface combatants of the Atlantic Fleet. For the calendar year 1983, 111 ships engage in 19 primary events yielding a model with 228 constraints and 10,723 binary variables. This model is solved optimally in about 1.6 minutes producing a schedule that is significantly better than the corresponding published schedule.
\end{abstract}

The optimized peacetime employment schedule which has as its objective maximizing combat readiness should always be the goal and guide.

U.S. Navy, NWP-I

He knew the things that were, the things that would be, and the things that had come before.

Homer, The Iliad

$\mathrm{T}$ he Atlantic Fleet Employment Schedule details the day-to-day operations of 700-750 ship, submarine, marine and air units. It consists of a directive quarterly schedule embedded within an annual schedule which is used for planning. The detailed quarterly schedule contains all tasks and activities to be conducted by fleet units while the annual planning schedule contains only major activities or events. This study is concerned with the annual planning schedule.

Requests for fleet units to participate in events originate from the Secretary of Defense, the Chief of Naval Operations, Type, Fleet, Group, Squadron and individual unit commanders, and others. This results in a complex employment scheduling problem which is made more complex because, typically, fleet assets are insufficient to meet all requests. Because of its complexity, current employment scheduling procedures demand the attention of operation and planning staffs at all levels in the command structure.

We seek to automate a substantial portion of the employment scheduling problem. Currently, no automated decision aids are used for producing the employment schedule. Rather, the employment schedule is a direct result of several quarterly, weeklong conferences involving Type Commanders and the Commander in Chief Atlantic Fleet (CINCLANTFLT).

Scheduling decisions directly affect fleet readiness and fleet operational performance. Unfortunately, readiness is a vague measure which cannot be directly optimized. However, an analytical method can be devised to assure that a good employment schedule provides the opportunity to maintain readiness at a high level. Assignment of a suboptimal mix of forces and capabilities to perform an operational mission or major exercise results in degraded performance and, in the extreme, may result in failure to achieve the objectives of the mission or exercise. Additionally, unnecessary or inequitable employment of fleet assets adversely affects personnel morale and reduces the opportunities for maintenance and training. While overemployment is considered more detrimental to fleet readiness, underemployment results in deficiencies in operational experience with a consequent reduction in overall readiness.

CINCLANTFLT has operational commitments in the home fleet (Second Fleet) and abroad. These commitments are principally expressed as a set of primary events, including all extended operations and major exercises-the most important and demanding events

Subject classifications: Military: force readiness. Programming: generalized set partitioning.

Operations Research

Vol. 38, No. 2, March-April 1990 
in the fleet schedule. Other events are classified as either major maintenance events or secondary events and may be viewed as events necessary to support the successful conduct of primary events.

We restrict the scope of our work by assuming that: 1) all primary events are fixed in start time and duration, 2) all primary events are uniformly more important than secondary events, and 3) all major maintenance events are fixed in start time and duration. Assumption 1 effectively separates the process of the timing of primary events from the problem of scheduling (assigning) units to these events. This is a good approximation of current practice because most commitments are made years in advance without detailed knowledge of future fleet assets, and also because of annually recurring commitments such as NATO exercises. Assumptions 2 and 3 allow assignment of units to primary events without requiring concomitant scheduling of supporting events, although time must be set aside in a unit's primary event schedule to allow for subsequent scheduling of secondary events. (Wing 1986 solves the "interdeployment scheduling" problem for secondary events.) Assumption 3 is true barring unforeseen equipment failures and results in known periods of operational availability for each fleet unit. Thus, with the above assumptions, the problems of determining which events to schedule and when to schedule them are presumed solved. The remaining problem is to determine which operationally available fleet units should be used to satisfy the primary event requirements while distributing the workload equitably among the units. We develop and implement a method of solving this problem.

The entire Atlantic Fleet employment scheduling problem is formidable. However, fleet units may be divided into the following highly independent functional categories: surface combatant, amphibious, marine, support, submarine, and aviation units. Within a category, unit operational capabilities are similar, and only within a category are units employed in similar missions; substitutions within a category may be allowed but substitutions across categories are not allowed. Thus, although primary events may require assets from one or more of the categories, an individual asset requirement for a primary event can only be satisfied from a single category. We develop a model for the Combatant Primary Event Schedule (CPSKED), which generates annual planning schedules for a single functional category, in this case, surface combatant ships.

The U.S.S. Spruance (DD 963), Figure 1, is a representative surface combatant. The Spruance is

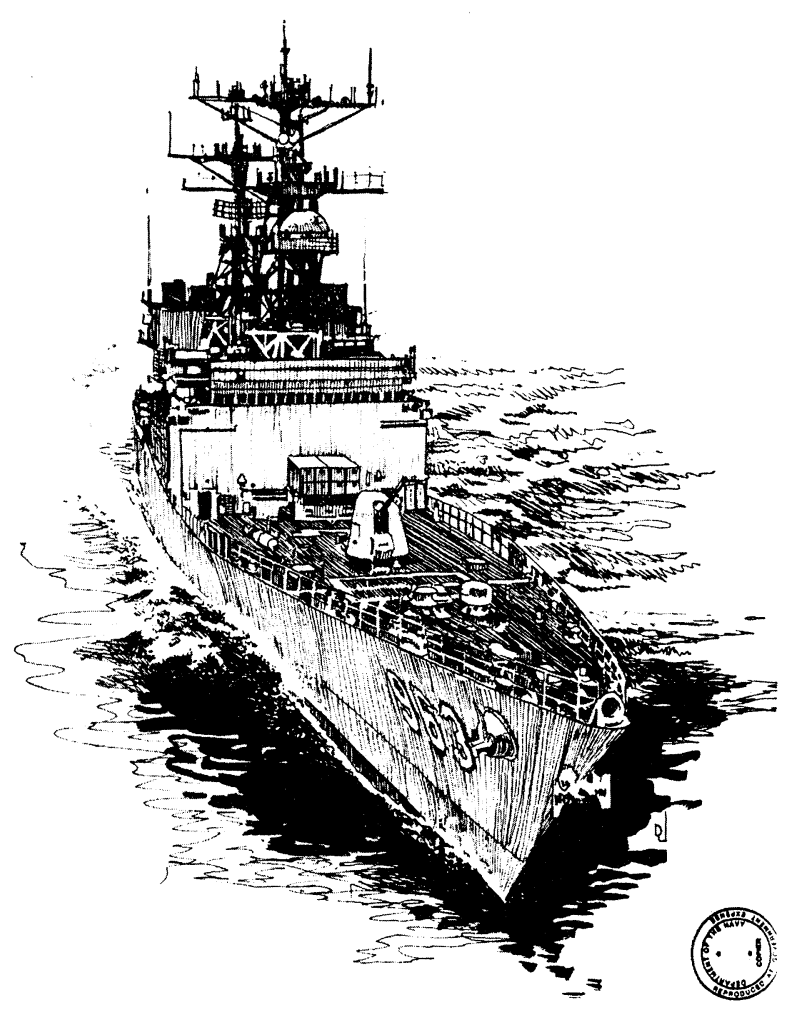

Figure 1. U.S.S. Spruance (DD 963), a representative surface combatant.

designed for antisubmarine warfare, especially as an integral part of larger task groups, and has a crew complement of 24 officers and 272 enlisted men. Propulsion by four gas turbines yields a range of 6,000 miles and a speed of 33 knots. Weapons systems include antisubmarine rockets and torpedoes, 5-inch guns, antiship and anti-air missiles, close-in-defense Gatling guns, and armed helicopters. Other systems include air, surface and fire-control radars, sonars, and fire-control computers, as well as a variety of tactical communications systems.

For surface combatants, primary event requirements can be broken down into two categories: requirements for a specific mix of ship types by event, and requirements for a specific mix of weapon systems by event. These requirements lead to problem constraints which are labeled event/ship-type requirements and event/weapon-system requirements. The goal of CPSKED is to assign ships to events to meet all event/ship-type requirements, to meet all event/weapon-system requirements, and to minimize the deviations from ideal schedules for individual ships.

Scheduling problems can sometimes be viewed as selection problems, for example, route selection 
(Brown, Graves and Ronen 1987), and crew selection (Marsten and Shephardson 1981). (However, ship scheduling problems can become too large for selection methods, or exhibit nonlinear interactions between schedules which defy concise linear modeling; for example, see Sibre (1977).) CPSKED assigns each candidate schedule a cost in terms of the deviation from an ideal schedule. Thus, our problem is to select a minimum cost set of candidate ship schedules such that demands for ship types and weapon systems required by all primary events are satisfied. Such a problem is amenable to formulation as a set partitioning problem, which we generalize to include equality, inequality and goal programming constraints. Goals are included because it may not be possible to meet event requirements at any cost as a result of insufficient assets.

The set partitioning approach to scheduling allows incorporation of many real-world modeling considerations; for example, sequencing constraints and objective coefficients that are nonlinear functions of individual schedules can be considered in the problem generator versus the problem solver. Other approaches can be unnecessarily restrictive. For instance, Appelgren (1969) solves a ship scheduling problem related to ours by using a Dantzig-Wolfe decomposition of a multicommodity flow formulation. That approach forces the use of objective coefficients that are linear functions of the individual ship schedules.

A solution approach is only good if available technology can solve the problems generated, and set partitioning problems belong to the difficult class of integer programming problems. Earlier work on solving similar models has met with considerable difficulty in achieving optimal integer answers (e.g., Appelgren 1969, 1971, Crawford and Sinclair 1977). However, recent technological advances make probable the solution of large problems (Bausch 1982, Brown, Graves and Ronen).

\section{SCHEDULING CRITERIA}

The Navy is directed to maximize national defense subject to constrained fleet resources. However, the value of each ship's employment schedule to the national defense cannot be measured in terms of dollars. Consequently, different measures of effectiveness must be developed for CPSKED.

The tasks and activities contained in the Employment Schedule are broken down into 27 categories which are further subdivided into specific employment terms (EMPTERMs) (U.S. Navy 1984a). We use the term event to refer to a collection of EMPTERMs related to the same task. For example, a deployment to the Mediterranean Ocean is an event that consists of the following EMPTERMs: EXER (a readiness exercise), POM (preparation for overseas movement), ENR (transit to the Mediterranean), OPCON (operational condition), ENR (transit back from the Mediterranean), and LVUPK (postdeployment leave and upkeep).

Primary events consist of extended operations and major exercises, deployment of a battle group to the Indian Ocean, or participation in a specific NATO exercise. These events have fixed start and completion times and form the backbone of the schedule.

Major maintenance events, for example, construction, conversion, and overhaul are dependent on shipyard availability and ship cycles. These events are generally scheduled independently of all other events. Units scheduled for major maintenance events are unavailable for conflicting primary events.

Secondary events include the remaining activities associated with maintenance, training, inspections and other individual ship events. Secondary events are viewed as preparation and support for the primary events and are scheduled not to conflict with the primary events.

A ship's employment cycle consists of the following phases: new construction or overhaul, operational, and refit (U.S. Navy 1983). A new cycle begins each time the ship enters overhaul. The operational phase consists of four periods: ready, preparation for overseas movement (POM), deployed, and postdeployment leave. During the ready period, the ship will participate in home-fleet operations and exercises. During the POM and post-deployment leave periods the ship remains in its home port. Any period in which the ship operates away from home port for more than eight weeks is considered a deployed period.

The scheduling objective stated by CINCLANTFLT policy (U.S. Navy 1984b) is to "... maintain the Fleet at the highest level of readiness for: 1) operations in the Atlantic; and 2) to ensure that individual units are fully ready for projected employment when deployed." This policy establishes “. . f firm scheduling criteria to provide for basic type training, allow for adequate ship maintenance, and ensure reasonable time in home port." Those policy guidelines that are pertinent to this study are summarized below:

a. Normally, not more than one third of the time between overhauls shall be committed to deployments. 
b. While in the home fleet, 10 days per quarter shall be available to each ship for the conduct of individual ship training.

c. Following extended operations, ships will be scheduled for a period of 15-30 days post deployment leave.

d. Ships will normally be assigned 20 working days of upkeep per quarter while in the home fleet.

e. Ships scheduled for extended operations will be scheduled for a POM period of three to four weeks duration just prior to deployment.

f. Ships in the operational phase will normally be scheduled for an optimum of 30 operating (at-sea) days per quarter while in the home fleet.

g. To the extent possible, employment schedules will provide each ship an average of $50 \%$ time in home port between overhauls.

Although not specified by CINCLANTFLT regulations, another major guideline is used by fleet schedulers:

h. Employment schedules will attempt to provide 360 days between successive deployments.

A schedule that provides the proper amount of homeport time for training, morale, and maintenance; the proper amount of home-fleet at-sea time for training; and an equitable deployment rotation of ships will provide the best opportunity to achieve the CINCLANTFLT goals for readiness.

Policies $\mathrm{c}$ and $\mathrm{e}$ are handled by extending the length of deployments to include pre- and post-deployment requirements. The other policy statements are used to derive ideal targets (goals) for each ship for time between deployments $T_{1}$ (policy $\mathrm{h}$ ), the ratio of deployed time to between-overhaul time $\tau_{2}$ (policy a), and the ratio of home-fleet sea time to home-fleet time $\tau_{3}$ (policies $\mathrm{b}, \mathrm{d}, \mathrm{f}$ and $\mathrm{g}$ ).

Home-fleet time consists of the operational phase time less deployment time. Home-fleet time is further broken down into the sum of home-fleet port time and home-fleet sea time. A good single measure of schedule effectiveness is a sum of weighted deviations from three target times, time between deployments $T_{1}$, deployed time $T_{2}$ and home-fleet sea time $T_{3}$, perhaps adjusted to encourage equity among ships. Targets $T_{2}$ and $T_{3}$ are derived from $\tau_{2}$ and $\tau_{3}$, respectively.

For the Employment Schedule as a whole, we must consider the real possibility that some of the event/ ship-type or event/weapon-system requirements cannot or should not be satisfied. CPSKED also treats these requirements as goals, with violation penalties included in the objective.

\section{MODEL FORMULATION}

The CPSKED problem is formulated first as a classical set partitioning problem generalized to include ranges on constraints, and then as an elastic set partitioning problem in which some constraints may be violated by incurring linear penalty costs. The objective function costs and penalties are developed in terms of the CINCLANTFLT policy previously described.

\section{Indices}

$k=1, \ldots, K$

(rows) constraints requiring that one schedule column be selected for each ship,

$i=1, \ldots, I$

(rows) event/ship-type requirements,

$l=1, \ldots, L$

(rows) event/weapon-system requirements,

$j=1, \ldots, J$

(columns) each represents an individual ship schedule,

$p=1, \ldots, P$

primary schedule events,

$q=1, \ldots, Q$

ship types,

$r=1, \ldots, R$

weapon-system types,

$I_{p q}$

index set for event/ship-type requirement $i$ belonging to event $p$, requiring ship type $q\left(\left|I_{p q}\right| \leqslant 1\right)$,

$L_{p r}$

index set for event/weapon-system requirement $l$ belonging to event $p$, requiring weapon system $r$ $\left(\left|L_{p r}\right| \leqslant 1\right)$,

$J_{k}$

index set for all schedule columns $j$ belonging to ship $k$.

\section{Given and Derived Data}

$c_{j}$

cost of schedule $j$,

$a_{k j}$

1 if schedule $j \in J_{k} ; 0$ otherwise,

$s_{i j}, \quad j \in J_{k}, \quad i \in I_{p q}$

1 if schedule $j$ assigns ship $k$ to event $p$ as ship-type $q ; 0$ otherwise,

$w_{l j}, \quad j \in J_{k}, \quad l \in L_{p r}$

1 if ship $k$ has weapon system $r ; 0$ otherwise,

$\underline{b}_{i}^{s}\left(\bar{b}_{i}^{s}\right), \quad i \in I_{p q}$ minimum (maximum) number of ships of type $q$ required for event $p$,

$\underline{b}_{l}^{w}\left(\bar{b}_{l}^{w}\right), \quad l \in L_{p r}$ minimum (maximum) weapon systems of type $r$ required for event $p$. 


\section{Decision Variables}

$x_{j}$

1 if schedule $j$ is selected; 0 otherwise.

\section{Classical Formulation}

Minimize $\sum_{j} c_{j} x_{k}$

subject to

$\sum_{j} a_{k j} x_{j}=1 \quad k=1, \ldots, K$

$\underline{b}_{i}^{s} \leqslant \sum_{j} s_{i j} x_{j} \leqslant \bar{b}_{i}^{s} \quad i=1, \ldots, I$

$\underline{b}_{l}^{w} \leqslant \sum_{j} w_{l j} x_{j} \leqslant \bar{b}_{l}^{w} \quad l=1, \ldots, L$

$x_{j} \in\{0,1\} \quad j=1, \ldots, J$.

Constraints (1) require that exactly one schedule per ship be selected. Constraints (2) put lower and upper ranges on the number of a particular ship type that may participate in an event. Constraints (3) put minimum and maximum ranges on the number of a particular weapon system that should be available for an event.

Because of limited fleet assets, it is not always possible to meet the constraints defined in the classical formulation of this problem. Necessary violations of constraints, at a cost, are accommodated in the following formulation where constraint ranges become goals that incur linear penalties when violated.

\section{Elastic Formulation}

Minimize

$$
\begin{aligned}
\sum_{j} c_{j} x_{j} & +\sum_{k} \underline{p}_{k}^{a} \underline{z}_{k}^{a}+\sum_{i}\left(\underline{p}_{i}^{s} \underline{z}_{i}^{s}+\bar{p}_{i}^{s} \bar{z}_{i}^{s}\right) \\
& +\sum_{l}\left(\underline{p}_{l}^{w} \underline{z}_{l}^{w}+\bar{p}_{l}^{w} \bar{z}_{l}^{w}\right)
\end{aligned}
$$

subject to

$$
\begin{array}{ll}
1-\underline{z}_{k}^{a} \leqslant \sum_{j} a_{k j} x_{j} \leqslant 1 & k=1, \ldots, K \\
\underline{b}_{i}^{s}-\underline{z}_{i}^{s} \leqslant \sum_{j} s_{i j} x_{j} \leqslant \bar{b}_{i}^{s}+\bar{z}_{i}^{s} & i=1, \ldots, I \\
\underline{b}_{l}^{w}-\underline{z}_{l}^{w} \leqslant \sum_{j} w_{l j} x_{j} \leqslant \bar{b}_{l}^{w}+\bar{z}_{l}^{w} & l=1, \ldots, L \\
x_{j} \in\{0,1\} & j=1, \ldots, J \\
\underline{z}_{k}^{a}, \underline{\underline{z}}_{i}^{s}, \bar{z}_{i}^{s}, \underline{z}_{l}^{w}, \bar{z}_{l}^{w} \geqslant 0 &
\end{array}
$$

where

$\underline{p}_{k}^{a} \quad$ penalty for not scheduling ship $k$, $\underline{p}_{i}^{s}\left(\bar{p}_{i}^{s}\right), i \in I_{p q}$

per unit penalty for assigning too few (too many) ships of type $q$ to event $p$, $\underline{p}_{l}^{w}\left(\bar{p}_{l}^{w}\right), l \in I_{p r}$

per unit penalty for assigning too few (too many) weapon systems of type $r$ to event $p$.

There is great leeway in defining the costs and penalties for the CPSKED model above. The primary requirement is that they yield results that are good by objective standards. The costs and penalties defined below yield good results and, in addition, are mutually consistent and intuitively appealing. However, other definitions might yield good results, too.

The costs for individual ship schedules are functions of the following three targets derived from CINCLANTFLT policy goals:

1. Achieve an ideal time $T_{1}$ between successive deployments for an individual ship.

2. Maintain an ideal ratio $\tau_{2}$ of ship's deployed time to between-overhaul time.

3. Maintain an ideal ratio $\tau_{3}$ of a ship's home-fleet sea time to home-fleet total time.

To obtain a schedule as close as possible to these targets for a ship $k$, we derive a cost structure for the model column costs $c_{j}, j \in J_{k}$, which measures deviations from these targets in a common measure, days. $T_{1}$, the time-between-deployment target, is given at 360 days. A deployed-time target in days, $T_{2}$, is computed from $\tau_{2}$, current employment cycle statistics and the time horizon. After a ship's deployed periods are determined for schedule $j$, a home-fleet sea-time target $T_{3}$ is derived from $\tau_{3}$, the home-fleet time in the schedule and other data. Weighted deviations from the three target times $T_{1}, T_{2}$ and $T_{3}$ are then computed for schedule $j$

$C_{h j}=\left\{\begin{array}{l}\bar{m}_{h} \times\left(\text { deviation above } T_{h}\right) \\ \underline{m}_{h} \times\left(\text { deviation below } T_{h}\right)\end{array}\right.$

for $h=1,2,3$, and where $\bar{m}_{h}$ and $\underline{m}_{h}$ are weights in the range $(0,1]$. In terms of CINCLANTFLT policy, it is more costly to overemploy rather than underemploy a unit. Consequently, the weights are chosen such that $\tilde{m}_{1}<\underline{m}_{1}, \bar{m}_{2}>\underline{m}_{2}$, and $\tilde{m}_{3}>\underline{m}_{3}$. (A more detailed description of this and other cost computations can be found in Goodman 1985).

The total deviation of a schedule $j$ is defined to be the sum of the individual deviations

$C_{j}=C_{1 j}+C_{2 j}+C_{3 j}$.

This measure is intuitively appealing because it can be viewed as a measure of the total weighted deviation 
in days from an ideal schedule for a particular ship. The sum of the total deviations over all ships is a measure of the deviation of the fleet employment schedule from an ideal schedule.

An acceptability factor $\alpha_{i j}$ is a measure of how well a substituting ship can perform the duties of a required ship type for a particular event and lies in the range 0 (not substitutable) to 1 (completely substitutable). If there are no substitutions in a ship schedule $j$, the overall acceptability is considered to be 1.0 and the total deviation for the column is as just described. If there are substitutions, the total deviation is adjusted by dividing by the average of the acceptability factors for the events contained in the schedule column

$$
C_{j}=\left(C_{1 j}+C_{2 j}+C_{3 j}\right) /\left(\sum_{i: s_{i j} \neq 0} \alpha_{i j} / \sum_{i} s_{i j}\right) \text {. }
$$

Though appealing, the adjusted total deviation model may result in poor decisions if used directly. This linear model does not distinguish between two ships, both having total deviations of $C$ (equitable) and the same two ships, together fulfilling the same event requirements, but one having total deviation 0 and the other $2 C$ (inequitable). To avoid this problem, we define the column cost to be $c_{j}=C_{j}^{2}$. This cost structure encourages the model to resolve ties by spreading the workload over a greater number of ships.

With costs computed for each candidate ship schedule, penalties for goal violations must then be computed. Since one schedule is desired for each ship, the lower ranges on the ship-schedule constraints (4) are set to 1. Violation of the lower range on a shipschedule constraint corresponds to not scheduling that ship. The lower penalty, then, should be the price at which it is acceptable to allow the ship to remain idle throughout the planning period. In the CPSKED model, the idle cost is computed for each ship and is equivalent to the column cost for a do-nothing column which is not otherwise included in the model.

The lower range $\underline{b}_{i}^{s}$ on an event/ship-type constraint corresponds to the minimum number of ships of a particular type required for the event. The lower penalty $p_{i}^{s}$ is a price above which the cost of committing additional assets to the event exceeds the value of the contribution of those assets. Since short exercises are less important than long deployments, $\underline{p}_{i}^{s}$ should be an increasing function of event length. We use a quadratic function of the event length to put the penalty value into the same units as the rest of the objective function.

Situations arise where a ship would be underemployed if all minimum event requirements $\underline{b}_{i}^{s}$ are met exactly. Under these circumstances, it may be desira- ble to schedule the ship for some events in excess of minimum event requirements in order to maintain training and proficiency for the ship. To allow for this possibility, the upper range $\bar{b}_{i}^{s}$ for all event requirements may be set above the minimum requirement, but low enough to avoid unwieldy events. The upper penalty $\bar{p}_{i}^{s}$, in effect when the upper range $\bar{b}_{i}^{s}$ is exceeded, is a quadratic function of the event length but weighted to be less than the lower penalty.

Many but not all primary events may require a specified set of force weapon-system capabilities. Weapon systems are not necessarily unique to ship types and hence, the event/weapon-system requirements may be satisfied by various mixes of ships. Penalties for violating these constraints are related to the additional value a particular weapon system contributes to an event's mission. These penalties should be high enough to enforce the constraints but less than event/ship-type penalties because a weapon system contributes less than an entire unit to the event's mission. The upper limits and penalties can be set in the model to avoid putting too many of a scarce weapon system in one event, but in this implementation the upper penalties were all set to 0 .

\section{PROBLEM GENERATION}

In the set partitioning formulation of the CPSKED problem, a variable and its corresponding coefficient matrix column represent a candidate schedule. A principal advantage of the set partitioning formulation is that intricate schedule constraints can be incorporated logically in the explicit column generator rather than mathematically in the set partition solver. To make the distinction clear, we use the term limitations to refer to the constraints incorporated in the column generator. Time restrictions, cost limits and other realworld limitations are used in the generator to produce only admissible schedules. However, if the limitations on admissibility of individual schedules are weak, or the time horizon is too long, too many candidate schedules will be created and the solver overwhelmed. Even when very strict limitations exist, the number of columns generated tends to increase exponentially with the time horizon. The key to successful column generation is choosing a reasonable time horizon and creating a column generator which incorporates as many of the schedule limitations as possible.

The following rules are used to determine whether or not a schedule for a particular ship is admissible:

Rule 1. A ship must be the proper type, or an allowable substitute, to satisfy an event/ship-type requirement. 
Rule 2. A ship may not participate in primary events when the unit is in a nonoperational status.

Rule 3. A ship cannot participate in more than one primary event at any one time.

These rules are used to generate all feasible ship schedules as follows.

For each ship $k$ perform the following steps:

Step 1. Determine the ship type $q$ and, using Rule 1 , select all events that require type $q$ units or allow type $q$ units as substitutions. This potential ship-event list is the list of events in which ship $k$ could potentially participate.

Step 2. Determine the ship's nonoperational periods from input data, and using Rule 2, delete from the potential ship-event list any event conflicting in time with nonoperational periods. The resulting list is the ship/event list.

Step 3. Construct a schedule network as follows: Define a dummy starting node $v_{0}$ and a node for each event in the ship-event list. Connect a directed arc from $v_{0}$ to all other nodes. Using Rule 3, connect an additional arc between every pair of event nodes which do not conflict in time, directing the arc from the earlier event to the later event.

Step 4. Let $v$ correspond to an event in a schedule. (The set of all directed $v_{0} \rightarrow v$ paths for all $v$ in the network corresponds to the set of all admissible schedules for the ship.) Enumerate each $v_{0} \rightarrow v$ path $j$ and specify column coefficients: a) $a_{k j}=1$; b) $s_{i j}=1$ if ship $k$ satisfies part of event/ship-type requirement $i$ on the $v_{0} \rightarrow v$ path; c) $w_{l j}=1$ if ship $k$ satisfies part of event/weapon-system requirement $l$ on the $v_{0} \rightarrow v$ path; and d) 0 otherwise.

Additional logical limitations can reduce problem size. Event requirement inputs may be specified by either general ship-type or ship hull number. When a scheduler knows a priori that a specific ship must participate in a certain event, the requirement is input by the ship's hull number; all columns for that ship are then restricted to contain that event. Similarly, if an event is only open to specific ships then only those ships will contain that event in their ship-schedule network, that is, only those units will be considered for satisfying the corresponding event/ship-type requirement. Thus, row and column reductions result from fixing assignments or restricting event participation.

Substitutions between ship types may be specified and allowed for each ship-type requirement. If there are $n$ of the required ship-type and $m$ of the substitution ship-type, then there will be $n+m$ candidates available to satisfy the requirement, and a consequential increase in the number of columns. Substitutions should be allowed sparingly and only where tactically feasible: for example, a carrier would never substitute for a frigate and a frigate would probably never substitute for a cruiser. Substitution strategy may have a dramatic effect on the number of columns generated.

The number of admissible columns produced is much less than the $O\left(2^{I}\right)$ combinations (all possible $0-1$ vectors of length $I$ ) that would be produced by a naive generator. Nevertheless, this number can grow very large. Many of these columns may correspond to unit schedules that severely over or underemploy the unit and are counterproductive to the maintenance of high fleet readiness.

Further significant reductions in the number of columns sent to the set partitioning solver are possible using a cost editing approach. After each schedule column is generated, the schedule's component and overall costs are computed. The column $j$ is then rejected if its costs violate any of the limits:

Maximum time-between-deployment cost $\bar{C}_{1}$ (reject if $C_{1 j}>\bar{C}_{1}$ ),

Maximum deployment cost $\bar{C}_{2}$ (reject if $C_{2 j}>\bar{C}_{2}$ ),

Maximum home-fleet sea cost $\bar{C}_{3}$ (reject if $C_{3 j}>\bar{C}_{3}$ ), and

Maximum schedule cost $\bar{C}$ (reject if $C_{j}>\bar{C}$ ).

If an event requires a specific ship by hull number, then that event becomes mandatory for the ship. Cost limits are ignored for any column that contains only mandatory events. Cost limits may have to be judiciously relaxed for particularly difficult employment schedules.

\section{IMPLEMENTATION AND RESULTS}

Test data for CPSKED have been extracted from the Atlantic Fleet projected annual schedule for calendar year 1983, the most contemporary unclassified example available at the time of this study. Our evaluation is based on comparisons of the CPSKED solutions with the actual CINCLANTFLT schedule.

CPSKED has been implemented in ANSI standard FORTRAN 77, compiled by IBM VS FORTRAN (1.4.1) with OPT $=3$ on an IBM 3033 AP computer, and run under the VM/CMS operating system. It consists of three parts: column generator, solver and report writer.

The problem generator reads easily edited files containing ship and event data. The solver is the X-System 
Table I

1983 Atlantic Fleet Combatants

\begin{tabular}{llc}
\hline & Type & Number \\
\hline Aircraft carriers & CV/CVN & 9 \\
Guided missile cruisers & CG/CGN & 14 \\
Guided missile destroyers & DDG & 23 \\
Destroyers & DD & 17 \\
Guided missile frigates & FFG & 19 \\
Frigates & FF & 29 \\
Total & & 111 \\
\hline
\end{tabular}

(Brown and Graves 1975). The report writer uses the ship data file, the event data file, and a schedule solution file to produce a Ship Statistic Report, Ship Schedules Report, and an Event Force Assignment Report.

The Atlantic Fleet carrier and surface combatant assets for the calendar year 1983 consisted of the ships listed in Table I. Nonoperational periods and other historical data for these assets are known and included in the ship data file. The requirement to select exactly one schedule for each ship results in 111 schedule selection constraints.

All extended operations and major exercises involving surface combatant units were extracted from the CINCLANTFLT annual schedule resulting in the event list displayed in Table II (listed in order of event start time).

A primary event is composed of a collection of subevents. Each of these subevents corresponds to an employment term (EMPTERM) used in the Atlantic Fleet Schedule. Each subevent is classified as deployed time, home-fleet sea time, or home-fleet import time.

Table II

1983 Primary Event List

\begin{tabular}{ll}
\hline Extended Operations & \multicolumn{1}{c}{ Major Exercises } \\
\hline MED 1-83 & COMPTUEX 2-83 \\
IO 1-83 & SOLID SHIELD 83 \\
MEF 1-83 & OCEAN SAFARI 83 \\
MEF 2-83 & COMPTUEX 3-83 \\
SNFL 1-83 & COMPTUEX 4-83 \\
IO 2-83 & COMPTUEX 1-84 \\
MED 2-83 & \\
MEF 3-83 & \\
SNFL 2-83 & \\
UNITAS & \\
MEF 4-83 & \\
IO 1-84 & \\
MED 1-84 & \\
\hline
\end{tabular}

Table III shows the subevents composing the primary event MED 2-83.

A primary event requires a specific force composition, with possible allowance for substitution of assets. These requirements result in the event/ship-type constraints. Typical requirements, based on the MED 2-83 example, are listed in Table IV.

Force weapon system requirements are based on current requirements for forces deploying to the Mediterranean, Middle East, and Indian Ocean. Typical requirements using the MED 2-83 example are listed in Table V. The 1983 primary events yield a total of 73 event/ship-type constraints and 44 event/ weapon-system constraints.

The parameters listed in Table VI represent the scheduling policy goals used in the model runs, and

Table III

MED 2-83 Subevents

\begin{tabular}{llcll}
\hline & & Start & End & Code \\
\hline Primary Event & MED 2-83 & 069 & 355 & \\
Subevents & EXER (Readex 1-83) & 069 & 092 & S \\
(EMPTERMs) & POM & 093 & 122 & I \\
& ENR (Transit) & 123 & 133 & D \\
& OPCON & 134 & 315 & D \\
& ENR (Transit) & 316 & 325 & D \\
& LVUPK (Stand down) & 326 & 355 & I \\
\hline
\end{tabular}

Codes: D, deployed time; I, home-fleet in port time; $\mathrm{S}$, homefleet sea time.

Table VII shows the parameters used for limiting column generation by cost.

The CINCLANTFLT annual schedule did not contain ship assignments for all primary events. UNITAS and several other exercises were scheduled with ship assignments indicated as to be determined. To place the CINCLANTFLT schedule on a comparable basis with CPSKED all known CINCLANTFLT ship assignments were fixed and CPSKED was run to optimize the remaining part of the schedule. Table VIII lists the schedule summary data used to compare the optimally completed CINCLANTFLT schedule to the optimal CPSKED schedule.

The CPSKED model yields a $70 \%$ improvement in quality and also violates fewer weapon system goals. Average individual unit costs are not only reduced, they are spread more equitably over the ships as indicated by reduced standard deviations.

A model can never capture all of the criteria involved in scheduling Navy ships and, consequently, 
Table IV

MED 2-83 Ship-Type Requirements

\begin{tabular}{lllc}
\hline \multicolumn{1}{c}{ Type } & Hull & Substitution & Number \\
\hline CV/CVN & 69 & None & 1 \\
CG/CGN & Any & DDG, $\alpha=0.7$ & 2 \\
DDG & Any & DD, $\alpha=0.8$ & 2 \\
DD & Any & None & 2 \\
FFG & Any & FF, $\alpha=0.7$ & 3 \\
FF & Any & None & 3 \\
\hline
\end{tabular}

Table V

MED 2-83 Weapon-System Requirements

\begin{tabular}{lc}
\hline \multicolumn{1}{c}{ System } & Number \\
\hline AAW Missile (SM-1/ER) & 2 \\
AAW Missile (SM-1/MR) & 4 \\
AAW Radar (SPS-48) & 3 \\
Data Link (NTDS) & 4 \\
Passive Sonar (TASS/TACTAS) & 3 \\
ASW Helicopter (LAMPS) & 3 \\
Guns (5 in./54) & 4 \\
\hline
\end{tabular}

the full magnitude of improvement indicated by this comparison may not be achieved. However, a human scheduler can neither evaluate all of the scheduling combinations considered by the model, nor can the scheduler hope to compute, in a timely fashion, measures of effectiveness with any precision. Significant scheduling improvements can be achieved by assisting the human scheduler with the model.

Further computational tests are typified in terms of problem characteristics, problem size, and model execution times. The model was run under the following conditions:

\section{CPSKED(NS)}

without substitutions but cost limits;

2. CPSKED(SU) with substitutions and no cost limits;

3. CPSKED(SL) with substitutions and cost limits (same as CPSKED in Table VIII);

\section{CPSKED $(\mathrm{CF})$}

with CINCLANTFLT fixed assignments (same as CINCLANTFLT in Table VIII).

Run 1 establishes a reference objective value and determines if all events can be satisfied without substitutions. Run 2 demonstrates the increase in problem size over run 1 caused by allowing substitutions and removing cost limits. Run 3 shows the reduction in problem size over run 2 which may be achieved by including cost limits in the problem generator. Run 4 gives the optimally completed CINCLANTFLT annual schedule. Results of the runs are summarized in Table IX.

The number of columns and nonzero elements sent to the solver is apparently a function of the number of substitutions allowed, the number of assignments fixed, the total number of constraints, and the cost limits imposed in the problem generator.

Substitutions dramatically increase the problem size as indicated by a comparison of runs 1 and 3 ; however, the event/ship-type penalties observed in run 1 indicate that all requirements could not be satisfied without substitutions. Commitments should be met, and consequently, substitutions are necessary to avoid event/ship-type penalties and event/weapon-system penalties. Comparing runs 2 and 3, the inclusion of cost limits in the problem generator results in a problem size reduction of approximately $30 \%$ with little degradation in the objective value. Fixing schedule assignments that are known a priori will significantly decrease the problem size; however, fixing assignments can be expected to increase costs and may increase the number of goal violations (compare runs 3 and 4). Solution times for these problems are relatively modest and compare favorably to solution times for other large-scale set partitioning problems (e.g., Bausch 1982, Brown et al. 1987, and Marsten and Shephardson 1981.

Table VI

Scheduling Policy Parameters

\begin{tabular}{lc}
\hline$T_{1}$ & 360 \\
$\tau_{2}$ & 0.33 \\
$\tau_{3}$ & 0.33 \\
$\underline{m}_{1}, \bar{m}_{2}, \bar{m}_{3}$ & 1 \\
$\bar{m}_{1}, \underline{m}_{2}$ & 0.1 \\
$\underline{m}_{3}$ & 0.25 \\
\hline
\end{tabular}

Table VII

Scheduling Parameters Limiting Costs

\begin{tabular}{lrrrrrr}
\hline & CV/CVN & CG/CGN & DDG & DD & FFG & \multicolumn{1}{c}{ FF } \\
\hline $\bar{C}_{1}$ & 120 & 120 & 120 & 120 & 120 & 120 \\
$\bar{C}_{2}$ & 180 & 180 & 120 & 120 & 90 & 90 \\
$\bar{C}_{3}$ & 90 & 90 & 60 & 60 & 45 & 45 \\
$\bar{C}$ & 300 & 180 & 150 & 150 & 120 & 120 \\
\hline
\end{tabular}


Table VIII

CINCLANTFLT vs. CPSKED

\begin{tabular}{|c|c|c|c|}
\hline & CINCLANTFLT & CPSKED & \% Improvement \\
\hline \multicolumn{4}{|l|}{ Objectives } \\
\hline Cost & $1,472,500$ & 446,700 & 70 \\
\hline \multicolumn{4}{|l|}{ Goal Violations } \\
\hline Schedule Selection & 0 & 0 & 0 \\
\hline Event/Ship Type & 0 & 0 & 0 \\
\hline Event/Weapon System & 11 & 9 & 18 \\
\hline \multicolumn{4}{|l|}{ Unit Costs Mean (std. dev.) } \\
\hline Total $\left(C_{j}\right)$ & $48.2(57.3)$ & $42.7(49.0)$ & $11(14)$ \\
\hline $\operatorname{TBD}\left(C_{1 j}\right)$ & 44.9 (43.9) & $34.7(28.9)$ & $23(34)$ \\
\hline $\operatorname{DEP}\left(C_{2 j}\right)$ & $25.9(40.4)$ & $24.5(40.1)$ & $5(1)$ \\
\hline $\operatorname{SEA}\left(C_{3 j}\right)$ & $3.9 \quad(4.4)$ & $2.9 \quad(3.3)$ & $26(25)$ \\
\hline \multicolumn{4}{|l|}{ Unit Statistics } \\
\hline TBD (target 360) & $329(142)$ & $344(103)$ & $5(27)$ \\
\hline DEP ratio (target 33 ) & $0.33(0.10)$ & $0.34(0.09)$ & $-3(10)$ \\
\hline SEA ratio (target 33) & $0.30(0.06)$ & $0.30(0.05)$ & $0(16)$ \\
\hline
\end{tabular}

Abbreviations: TBD, time between deployments; DEP, deployed time; SEA, home-fleet sea time.

Table IX

CPSKED Results

\begin{tabular}{|c|c|c|c|c|}
\hline & $\begin{array}{c}\text { Run } 1 \\
\text { CPSKED(NS) }\end{array}$ & $\begin{array}{c}\text { Run } 2 \\
\text { CPSKED(SU) } \\
\end{array}$ & $\begin{array}{c}\text { Run } 3 \\
\text { CPSKED(SL) }\end{array}$ & $\begin{array}{c}\text { Run } 4 \\
\text { CPSKED(CF) }\end{array}$ \\
\hline \multicolumn{5}{|l|}{ Characteristics } \\
\hline Total Ships & 111 & 111 & 111 & 111 \\
\hline Total Events & 19 & 19 & 19 & 19 \\
\hline Substitutions Allowed & No & Yes & Yes & Yes \\
\hline Cost Limits & Yes & No & Yes & Yes \\
\hline \multicolumn{5}{|l|}{ Problem Size } \\
\hline Rows & 228 & 228 & 228 & 228 \\
\hline Columns & 4,109 & 15,193 & 10,723 & 3,984 \\
\hline Nonzeros & 19,019 & 84,247 & 55,404 & 19,092 \\
\hline \multicolumn{5}{|l|}{ Run Times (in cpu seconds) } \\
\hline Generator & 2.3 & 8.3 & 6.2 & 2.4 \\
\hline Solver & 63.2 & 131.2 & 97.8 & 59.4 \\
\hline Reports & 0.6 & 0.7 & 0.7 & 0.7 \\
\hline \multicolumn{5}{|l|}{ Objectives } \\
\hline Cost & 395,200 & 427,000 & 446,700 & $1,472,500$ \\
\hline \multicolumn{5}{|l|}{ Goal Violations } \\
\hline Schedule Selection & 0 & 0 & 0 & 0 \\
\hline Event/Ship Type & $4,144,000$ & 0 & 0 . & 0 \\
\hline Event/Weapon System & 10,000 & 7,000 & 9,000 & 11,000 \\
\hline Total & $4,549,200$ & 434,000 & 455,700 & $1,483,500$ \\
\hline
\end{tabular}

\section{CONCLUSION}

The CPSKED versus CINCLANTFLT schedule comparisons indicate that fleet employment schedules can be improved. Optimization techniques can efficiently produce good annual fleet employment schedules. Response times are short enough to permit using this model in an interactive schedule planning system, and refinements in our prototypic implementation of this model can further improve performance.
An optimization model provides a means for objectively considering all alternatives to determine the best schedule subject to the constraints supplied. This schedule may then be used as a reference for comparing alternative schedules that may include additional criteria not evident or even ponderable in the initial model run. This process may be conducted iteratively until a final acceptable annual schedule is developed. The optimization model ensures that objective model costs are minimized. The scheduler must decide 
whether the additional subjective criteria are justifiable in terms of the resulting increased costs. Thus, the model provides the decision maker with the capability of producing high quality optimum schedules that satisfy, or at least consider, all scheduling criteria. CPSKED is a powerful management tool for developing, refining and maintaining employment schedules.

\section{ACKNOWLEDGMENT}

Dan Bausch synthesized a host of promising alternatives for solving set partitioning problems into a concise canonical strategy, and showed which ones worked. Glenn Graves has always backed us with ready computational advice and support.

\section{REFERENCES}

Appelgren, L. H. 1969. A Column Generation Algorithm for a Ship Scheduling Problem. Trans. Sci. 3, 53-68.

APPelgRen, L. H. 1971. Integer Programming Methods for a Vessel Scheduling Problem. Trans. Sci. 5, 64-78.

BausCH, D. O. 1982. Computational Advances in the Solution of Large-Scale Set Covering and Set Partitioning Problems. M.S. Thesis, Operations Research Department, Naval Postgraduate School, Monterey, Calif.

Brown, G. G., and G. W. Graves. 1975. Elastic Pro- gramming: A New Approach to Large-Scale Mixed Integer Optimization. Paper Presented at the ORSA/ TIMS Meeting, Las Vegas.

Brown, G. G., G. W. Graves and D. Ronen. 1987. Scheduling Ocean Transportation of Crude Oil. Mgmt. Sci. 33, 335-346.

Crawford, J. L., AND G. B. Sinclair. 1977. Computer Scheduling of Beer Tanker Deliveries. Int. J. Phys. Dist. 7, 294-304.

Goodman, C. E. 1985. Annual Scheduling of Atlantic Fleet Naval Combatants. M.S. Thesis, Operations Research Department, Naval Postgraduate School, Monterey, Calif.

Marsten, R. E., and F. Shephardson. 1981. Exact Solution of Crew Scheduling Problems Using the Set Partitioning Model: Recent Successful Applications. Networks 11, 165-177.

Sibre, C. E. 1977. A Quadratic Assignment/Linear Programming Approach to Ship Scheduling. M.S. Thesis, Operations Research Department, Naval Postgraduate School, Monterey, Calif.

U.S. Navy. 1983. Strategic Concepts of the U.S. Navy. Chief of Naval Operations, Naval Warfare Publication NWP-1.

U.S. Navy. 1984a. Operational Reports (Revision A). Chief of Naval Operations, Naval Warfare Publication NWP-7.

U.S. Navy. 1984b. CINCLANTFLT 5400.2J. Atlantic Fleet Regulations. Commander in Chief, U.S. Atlantic Fleet.

WING, V. F. 1986. SURFSKED: An Optimization Aid for Surface Combatant Inter-Deployment Scheduling. M.S. Thesis, Operations Research Department, Naval Postgraduate School, Monterey, Calif. 